\title{
Speech Intelligibility Measurements in Tinnitus Patients with and without Hearing Loss
}

\author{
A. Wicher ${ }^{a}$, E. OzIMEK ${ }^{a}$ AND E. SzYMieC ${ }^{b *}$ \\ ${ }^{a}$ Institute of Acoustics, A. Mickiewicz University, Umultowska 85, 61-614 Poznań, Poland \\ ${ }^{b}$ ENT Department, Karol Marcinkowski University of Medical Sciences, Fredry 10, 61-701 Poznań, Poland \\ The aim of the study was to determine the effect of hearing loss on speech intelligibility in tinnitus patients. \\ Two new Polish tests for speech intelligibility measurements (the Polish Sentence Test-PST and the Polish Digit \\ Triplet Test-PDTT) were used in the investigation. Tinnitus patients (56 persons) with bilateral sensorineural \\ hearing loss and 28 tinnitus patients with normal hearing thresholds participated in the study. For tinnitus patients \\ with hearing loss, two slopes of the audiograms for higher frequencies were chosen. The speech reception threshold \\ $(\mathrm{SRT})$ for sentence test $\left(\mathrm{SRT}_{\mathrm{S}}\right)$ and for triplet test $\left(\mathrm{SRT}_{\mathrm{T}}\right)$ were determined. Results showed that for tinnitus \\ patients (without hearing loss), the mean $\mathrm{SRT}_{\mathrm{S}}=-4.2 \mathrm{~dB}$ and was higher by $-1.9 \mathrm{~dB}$ than that for normal hearing \\ subjects (control group). For the triplet test, the mean $\mathrm{SRT}_{\mathrm{T}}=-8.1$, and was by $-1.3 \mathrm{~dB}$ higher than $\mathrm{SRT}_{\mathrm{T}}$ \\ for the control group. This meant that the PST reflected a greater tinnitus influence on the speech intelligibility \\ than the PDTT. The mean $\mathrm{SRT}_{\mathrm{S}}$ values for tinnitus patients with sloping audiogram up to $15 \mathrm{~dB} /$ oct were within \\ the range from -3.2 to $-0.7 \mathrm{~dB}$, while for the larger slopes the mean $\mathrm{SRT}_{\mathrm{S}}$ values changed from $-2.2 \mathrm{~dB}$ to $0.48 \mathrm{~dB}$.
}

PACS numbers: 43.71.-k, 43.71.Gv, 43.71.Ky

\section{Introduction}

Tests used to evaluate speech intelligibility are generally divided according to the mode of presentation of the linguistic material, i.e. the speech can be presented in silence or against a background of disturbing noise. A further division of the tests is related to the type of linguistic material used in the test. There are sentence tests [1-5], word tests [6-8] or digital and numerical tests [9-13] used to measure speech intelligibility. According to diagnostic possibilities the tests can be divided into screening tests used for preliminary evaluation of speech intelligibility and tests of clinical applications for detailed diagnostics of the auditory system.

Recently, within the European Framework Project 6 "Hearing in Communication Society" (acronym HearCom), at the Institute of Acoustics A. Mickiewicz University, two new tests were developed for speech intelligibility against a background of disturbing noise: the Polish Sentence Test (PST) [3] and the Polish Digit Triplet Test (PDTT) $[9,10]$. PST belongs to the tests used for clinical and diagnostic purposes, while PDTT is used for screening studies.

The main purpose of the study was to determine the effect of tinnitus and hearing loss on intelligibility of speech presented against a background noise. The use of the speech intelligibility tests in examination of sub-

* corresponding author; e-mail: eszymiec@poczta.onet.pl jects with hearing loss permits determination of the relation between the type and degree of hearing loss and the speech reception threshold (SRT). The latter parameter is the signal to noise ratio at which the speech is understood in 50\%. Literature on perception studies for hearing impaired subjects proposes many ways of classification of the hearing loss degree. Beutelmann and Brand [14] presented a detailed classification of hearing loss degree taking into account the mean hearing loss (determined as the mean hearing threshold for the frequencies of $0.5,1,2$ and $4 \mathrm{kHz}$ ) and the shape of the audiogram (flat, sloping or notch-shaped). In the majority of studies the classification of hearing loss is related only to determination of the mean hearing threshold for a few frequencies in the range of 0.25 to $6 \mathrm{kHz}[15,16]$. The incorrect functioning of the auditory system can be manifested not only as hearing loss but also as tinnitus, i.e. the hearing sensation appearing without the source of sound and related to pathologies in the inner ear or at higher levels of the auditory pathway [17].

This study was performed with the participation of tinnitus patients divided into two groups. The first group included patients with tinnitus and without hearing loss, while the second group comprised the patients with tinnitus and different degree of hearing losses.

\section{Apparatus and measurement procedure \\ 2.1. Apparatus and method}

The sentence test consists of 37 lists of 13 sentences each. This test was phonemically and statistically bal- 
anced, i.e. the respective lists revealed comparable phonemic content and similar psychometric functions. All sentences were taken from everyday speech, literature, TV and theatre. All of them fulfilled the fundamental definition of a sentence, i.e. they had a subject, a verb and an object. They contained normal everyday contexts. The following criteria were used in the selection of the sentences $[3,5,10]$ : the total number of syllables in a sentence should be equal to eight or nine (number of words ranging from 3 to 7 ; mean 4.6 words/per sentence); the words in the sentences should not contain more than three syllables each; the sentences should not contain punctuation characters and capitals (excluding the initial capital). No duplicate sentences were selected. The sentences should be grammatically and syntactically correct and semantically neutral, which excluded political, war or gender-related topics. Questions, proverbs, proper names and exclamations were eliminated. The Digit Triplet Test is composed of four statistically balanced lists. Each of them contains 25 different digit triplets $[7,9]$. The digit triplet is a complex of three digits (for example 2-4-7, pronounced separately as two-four-seven).

The intelligibility measurements were carried out using the standard adaptive procedure with the 1-up/1-down decision rule, i.e. after a correct or incorrect response given by the subject, the SNR was either decreased or increased. The subject's response was considered correct when all the words constituting the presented sentence and their order were repeated correctly. In such case the score was $100 \%$. Otherwise, the score was set to $0 \%$. The initial signal-to-noise ratio (SNR) was set to $-2 \mathrm{~dB}$, i.e. the speech was relatively easily understood at the beginning of the measurement. The initial step was $2 \mathrm{~dB}$ and was reduced to $1 \mathrm{~dB}$ after the first incorrect response. The SRT was computed as the mean of the last $22 \mathrm{SNR}$ values (for two randomly chosen lists). Similar procedures were used for PDTT, applying one of the four randomly selected triplet lists. The application for speech intelligibility measurements was installed on the PC computer equipped with an external Sound Blaster $\mathrm{X}-\mathrm{Fi}$ sound card. The signals were transmitted to the Interacoustics AC 40 audiometer and were presented to patients monaurally via the TDH 39 headphones.

\subsection{Patients}

Tinnitus patients ( 56 persons) who exhibited bilateral sensorineural hearing losses and 28 tinnitus patients with normal hearing thresholds participated in the study. All patients were selected form the Centre of Laryngological Rehabilitation, Medical University in Poznań. The age of the tinnitus patients with normal hearing thresholds ranged from 20 to 71 years, median $=41$ years, quartiles $=23$ and 38 years, and in the case of hearing impaired patients with tinnitus the age ranged from 27 to 82 years, median $=60$ years and quartiles $=54$ and 70 years.

Before speech intelligibility data were collected, middle-ear testing by tympanometry measurements and clinical pure-tone audiometry had been performed. Results of the tympanometry measurements showed that for all patients the middle ear transmission systems were normal according to routine clinical measures.

Hearing impaired patients with tinnitus had sloping hearing loss functions characterised by two values of slopes of the audiograms for higher frequencies. They were divided into three groups on the basis of the average pure-tone threshold (PTT) at $0.25,0.5$ and $1 \mathrm{kHz}$ $\left(\mathrm{PTT}_{0.25,0.5,1}\right)$ : (1) $\mathrm{PTT}_{0.25,0.5,1}$ up to $20 \mathrm{~dB} \mathrm{HL},(2)$ $\mathrm{PTT}_{0.25,0.5,1}$ in the range of $20-30 \mathrm{~dB} \mathrm{HL}$ and (3) $\mathrm{PTT}_{0.25,0.5,1}$ above $30 \mathrm{~dB} \mathrm{HL}$. Additionally, in each of these groups two values of slopes of the hearing loss functions in the range of $1-4 \mathrm{kHz}$ were distinguished: (1) up to $15 \mathrm{~dB} /$ oct and (2) above $15 \mathrm{~dB} /$ oct.

\section{Results}

Prior to the speech intelligibility tests, the tinnitus parameters were determined: the frequencies corresponding to the perceived tinnitus and the minimum masking level (ML), i.e. the minimum level of external noise that would mask the tinnitus perceived by the patient. Two types of tinnitus were distinguished: tonal and noise bands of $1 / 3$ octave width. The tinnitus was evaluated by comparing the tinnitus perceived by the subject with the signal generated by the audiometer (tone or $1 / 3$ octave noise band) [17]. In the patients without hearing loss the tinnitus created hearing sensation similar to that of a noise band. $62 \%$ of patients with hearing loss perceived tinnitus of the tonal character and $38 \%$ of them perceived it as $1 / 3$ octave noise. Figure 1 presents the percentage frequency distribution of the tinnitus perceived by the subjects without hearing loss (1A) and that perceived by the subjects with hearing loss (1B). The tinnitus of the frequencies of 250 and $500 \mathrm{~Hz}$ was found in $60 \%$ of the patients without hearing loss (Fig. 1A), whereas the tinnitus of the frequencies in the range of $4-8 \mathrm{kHz}$ was established in $77 \%$ of the subjects with hearing loss. It should be mentioned that in the tinnitus patients without hearing loss the tinnitus perceived was similar to the $1 / 3$ octave noise band, while that in the subjects with hearing loss was close to tones in the majority of cases and close to noise bands in the rest.

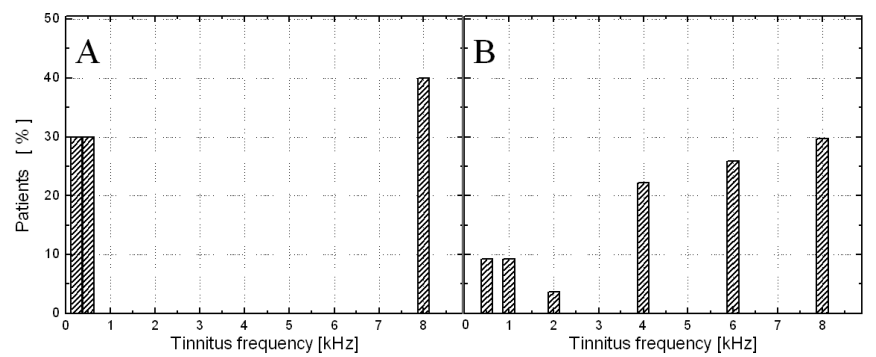

Fig. 1. Distributions of the tinnitus frequency for patients with normal hearing thresholds (panel A) and for hearing impaired patients (panel B). 
Figure 2 presents the percentage distribution of the minimum masking level of the perceived tinnitus for subjects without hearing loss (Fig. 2A) and for subjects with hearing losses (Fig. 2B). In both groups of subjects the ML value usually did not exceed $5 \mathrm{~dB}$ SL. Only for a few subjects with hearing loss the ML values were greater, 5-10 dB SL. This means that the tinnitus perceived by a given person will be masked by a signal of the same frequency or the middle frequency (of the noise band) and at a value of a few ten $\mathrm{dB}$ above the hearing threshold. These results are in agreement with the data on the analysis of tinnitus reported by other authors [17].

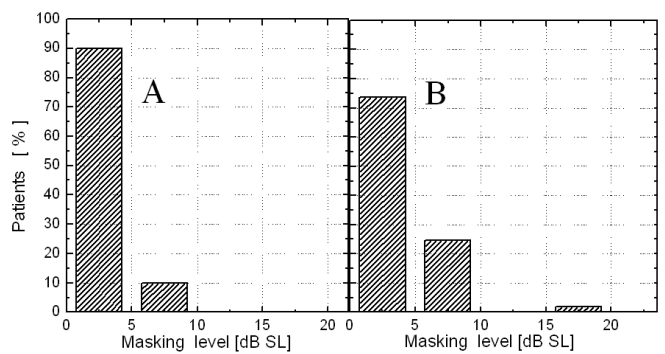

Fig. 2. Distributions of the tinnitus masking level for patients with normal hearing thresholds (panel A) and for hearing impaired patients (panel B).

The subjects taking part in the study were divided into groups according to the mean hearing threshold and slopes of the audiograms (cf. 2.2). Figure 3 presents the mean audiograms for the patients with tinnitus and without hearing loss (Fig. 3A), for the subjects with tinnitus and with the hearing loss $\mathrm{PTT}_{0.25,0.5,1}$ not exceeding $20 \mathrm{~dB}$ HL (Fig. 3B), those of $\mathrm{PTT}_{0.25,0.5,1}$ in the range 20-30 dB HL (Fig. 3C), and those of $\mathrm{PTT}_{0.25,0.5,1}$ above $30 \mathrm{~dB}$ HL (Fig. 3D).

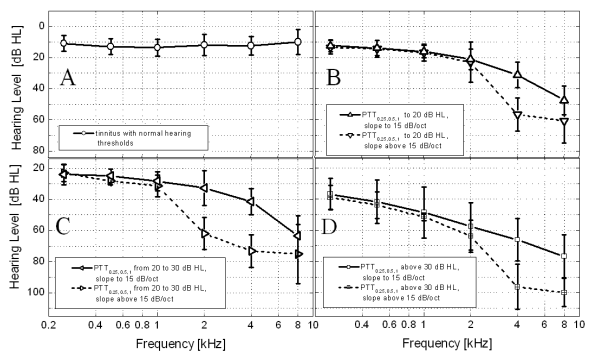

Fig. 3. Audiograms averaged across patients They were divided into four groups: (A) tinnitus patients with normal hearing thresholds, (B) $\mathrm{PTT}_{0.25,0.5,1}$ up to $20 \mathrm{~dB}$ HL, (C) $\mathrm{PTT}_{0.25,0.5,1}$ in the range of $20-30 \mathrm{~dB}$ HL and (D) $\mathrm{PTT}_{0.25,0.5,1}$ above $30 \mathrm{~dB}$ HL. Additionally, in each of $\mathrm{B}, \mathrm{C}$ and $\mathrm{D}$ panels two values of slopes of the hearing loss functions in the range of $1-4 \mathrm{kHz}$ were distinguished: (1) up to $15 \mathrm{~dB} /$ oct and (2) above $15 \mathrm{~dB} /$ oct.

The vertical bars in the audiograms represent standard deviation. The audiogram functions were divided according to the slope (solid line - the slope up to $15 \mathrm{~dB} /$ oct, broken line - the slope above $15 \mathrm{~dB} /$ oct) in the frequency range $1-4 \mathrm{~Hz}$ (Fig. 3B-D). The main objective was to determine the speech reception threshold for the sentence test $\left(\mathrm{SRT}_{\mathrm{S}}\right)$ and for the triplet test $\left(\mathrm{SRT}_{\mathrm{T}}\right)$. Figure 4 presents the relation between the mean speech reception threshold for the triplet test and the mean speech reception threshold for the sentence test.

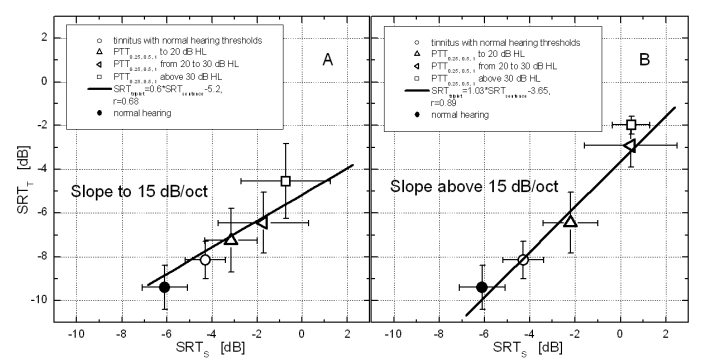

Fig. 4. Mean $\mathrm{SRT}_{\mathrm{T}}$ versus mean $\mathrm{SRT}_{\mathrm{S}}$ for different hearing losses together with function of the linear regression. Additionally, mean SRT values for normal hearing subjects are depicted.

Figure 4A presents the data obtained for patients with hearing loss whose audiogram slopes in the $1-4 \mathrm{kHz}$ range were smaller than $15 \mathrm{~dB} /$ oct, while Fig. $4 \mathrm{~B}$ presents the analogous data for patients whose audiogram slopes were greater than $15 \mathrm{~dB} /$ oct. Moreover, the SRT for normal hearing patients taken from Refs. [3, 9, 10] (full circles) and the SRT for subjects with tinnitus without hearing loss (empty circles) are marked. The vertical and horizontal bars stand for standard deviation. The correlation between SRT obtained in the two tests was checked, the correlation coefficient $r$ and the linear regression equation were determined. The linear regression functions are depicted in Fig. 4 as solid lines. The results for normal hearing patients were not taken into account in the analysis of regression; these data are only shown for reference.

\section{Discussion}

The use of new intelligibility tests (PST and PDTT) for estimation of speech ineligibility made it possible to determine mutual relations between their SRT values. Preliminary hearing diagnostic of the patients allowed identification of one type of hearing loss described by the same origin (cochlear, sensorineural hearing loss) and the same shape of the audiogram (sloping). Analysis of tinnitus included determination of their parameters such as type (tone, noise), frequency and masking level. In subjects without hearing loss the tinnitus most often refers to low frequencies (Fig. 1A), while in patients with hearing loss tinnitus involves mainly high frequencies (Fig. 1B). The threshold of tinnitus masking usually did not exceed 5 dB SL (Fig. 2). Taking into consideration low frequencies of tinnitus in patients without hearing loss it is reasonable to expect that tinnitus influences the perception of speech whose frequency components are mainly 
contained in the band up to $2 \mathrm{kHz}[3,9]$. On the other hand, the test material was presented at the comfortable level of hearing so tinnitus was masked, which theoretically could eliminate the effect of tinnitus on speech perception. However, it should be noted that the presence of tinnitus is related to pathologies at a higher auditory pathway. As proved in [17] the presence of tinnitus in patients without hearing losses is related to a statistically significant decrease in the otoacoustic emission, which means deteriorated functioning of the sensorineural part of the hearing system and can contribute to the problems with speech intelligibility.

A classification of the hearing losses according to the mean threshold of hearing for the frequencies 250, 500 and $1000 \mathrm{~Hz}\left(\mathrm{PTT}_{0.25,0.5,1}\right)$ and according to the slope of the audiogram for the frequencies $1-4 \mathrm{kHz}$ has been proposed. Figure 3 presents the mean audiograms showing mean hearing thresholds and mean sloping. This classification has been taken into account in determination of the mean values of $\mathrm{SRT}_{\mathrm{S}}$ and $\mathrm{SRT}_{\mathrm{T}}$. The relations between the speech reception threshold for the sentence test, the speech reception threshold for the triplet test and the changes in hearing loss as well as the slope of the audiogram can be approximated by linear dependence (Fig. 4). With increasing slope of the audiogram the slope of the function of the linear regression corresponding to the relation between SRT values for the two tests increases. The correlation coefficients $r$ indicate a stronger linear correlation between $\mathrm{SRT}_{\mathrm{S}}$ and $\mathrm{SRT}_{\mathrm{T}}$ for steeper audiogram functions. It also means that the increase in the hearing loss for the frequencies above $1 \mathrm{kHz}$ leads to increased values of SRT for both tests. The mean $\mathrm{SRT}_{\mathrm{S}}$ for tinnitus patients with hearing loss and the audiogram slopes up to $15 \mathrm{~dB}$ /oct are in the range of -3.2 to $-0.7 \mathrm{~dB}$, whereas for greater slopes, $\mathrm{SRT}_{\mathrm{S}}$ takes values in the range of $-2.2 \mathrm{~dB}$ to $0.48 \mathrm{~dB}$. The increase in the audiogram function causes a shift of the range of the mean $\mathrm{SRT}_{\mathrm{S}}$ by $1 \mathrm{~dB}$. For the triplet test, an increase in the slope of the audiogram function causes a change in the range of the mean $\mathrm{SRT}_{\mathrm{T}}$ by $2 \mathrm{~dB}$.

For subjects with tinnitus and without hearing loss, the mean $\mathrm{SRT}_{\mathrm{S}}$ is $-4.2 \mathrm{~dB}$, which is by $-1.9 \mathrm{~dB}$ greater than for those with normal hearing. For the triplet test the mean $\mathrm{SRT}_{\mathrm{T}}$ is $-8.1 \mathrm{~dB}$ and higher by $-1.3 \mathrm{~dB}$ than the value for the control group. This means that the results of the sentence test better characterise the deterioration in speech intelligibility in tinnitus subjects than those of PDTT. Therefore, the sentence test should be used for determination of the effect of hearing loss on the speech reception threshold. The triplet test being of the screening type permits only a preliminary evaluation of speech intelligibility. However, the triplet test results for a specific type of hearing loss help draw conclusions about the SRT of the sentence test. At present, further studies on speech intelligibility for hearing impaired subjects without tinnitus and audiograms of different shapes are underway. These results will make it possible to determine the effect of tinnitus on speech intelligibility in hearing impaired persons.

\section{Acknowledgments}

This paper was supported by the grant from the European Union FP6, Project 004171 HEARCOM, the State Ministry of Education and Science and Polish-Norwegian Research Fund.

\section{References}

[1] B. Kollmeier, M. Wesselkamp, J. Acoust. Soc. Am. 102, 1085 (1997).

[2] M. Nilsson, S.D. Soli, J.A. Sullivan, J. Acoust. Soc. Am. 95, 1085 (1994).

[3] E. Ozimek, D. Kutzner, A. Sęk, A. Wicher, Int. J. Audiology 48, 440 (2009).

[4] R. Plomp, A.M. Mimpen, Audiology 18, 43 (1979).

[5] N.J. Versfeld, L. Daalder, J.M. Festen, T. Houtgast, J. Acoust. Soc. Am. 107, 1671 (2000).

[6] A.J. Bosman, G.F. Smoorenburg, Audiology 34, 260 (1995).

[7] A. Pruszewicz, G. Demenko, L. Richter, T. Wika, Otolaryngol. Pol. 48, 56 (1994).

[8] A. Pruszewicz, G. Demenko, L. Richter, T. Wika, Otolaryngol. Pol. 48, 50 (1994).

[9] E. Ozimek, D. Kutzner, A. Sęk, A. Wicher, Speech Comm. 51, 307 (2009).

[10] E. Ozimek, D. Kutzner, A. Sęk, A. Wicher, Arch. Acoust. 32, 179 (2007).

[11] C. Smits, T. Houtgast, Int. J. Audiol. 46, 134 (2007).

[12] C. Smits, T. Kapteyn, T. Houtgast, Int. J. Audiol. 43, 15 (2004).

[13] K. Wagener, F. Eeenboom, T. Brand, B. Kollmeier, Ziffer-Tripel-Test: Spracherverstandlichkeitstest uber das Telefon, Tagungs- CD der DGA Jahrestagung (2005).

[14] R. Beutelmann, T. Brand, J. Acoust. Soc. Am. 120 , 331 (2006).

[15] E.L.J. George, A.A. Zekveld, S.E. Kramer, S.T. Goverts, J.M. Festen, T. Houtgast, J. Acoust. Soc. Am. 121, 2362 (2007).

[16] R.H. Wilson, R.A. McArdle, S.L. Smith, J. Speech. Lang. Hear. Res. 50, 844 (2007).

[17] E. Ozimek, A. Wicher, W. Szyfter, E. Szymiec, J. Acoust. Soc. Am. 119, 527 (2006).

[18] W.D. Hanks, G.D. Johnson, J. Speech. Lang. Hear. Res. 41, 1335 (1998). 